
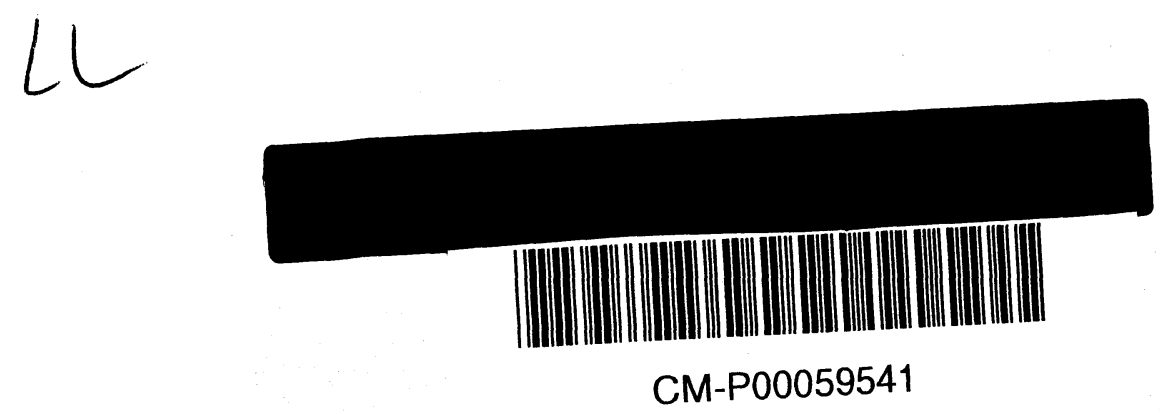

CERN/PS 93-47 (OP)

$$
\operatorname{sic} 9348
$$

9

CONDITIONING OF HIGH VOLTAGE RADIO FREQUENCY CAVITIES BY USING FUZZY LOGIC IN CONNECTION WITH RULE BASED PROGRAMMING.

S.Perreard*, E.Wildner

* Ecole des Mines de Saint-Etienne, France

* Ecole des Mines de Saint-Etienne, France

International Conference on Accelerator and Large Experimental Physics Control Systems

Berlin, Germany, October 18-22, 1993

Geneva, Switzerland

15/11/93 


\title{
CONDITIONING OF HIGH VOLTAGE RADIO FREQUENCY CAVITIES BY USING FUZZY LOGIC IN CONNECTION WITH RULE BASED PROGRAMMING.
}

\author{
S.Perreard*, E.Wildner** \\ * Ecole des Mines de Saint-Etienne, France \\ ** PS Division, CERN, CH-1211 Geneva, Switzerland
}

Many processes are controlled by experts using some kind of mental model to decide actions and make conclusions. This model, based on heuristic knowledge, can often be conveniently represented in rules and has not to be particularly accurate. This is the case for the problem of conditioning high voltage radio-frequency cavities: the expert has to decide, by observing some criteria, if he can increase or if he has to decrease the voltage and by how much.

A program has been implemented which can be applied to a class of similar problems. The kernel of the program is a small rule base, which is independent of the kind of cavity. To model a specific cavity, we use fuzzy logic which is implemented as a separate routine called by the rule base. We use fuzzy logic to translate from numeric to symbolic information.

The example we chose for applying this kind of technique can be implemented by sequential programming. The two versions exist for comparison. However, we believe that this kind of programming can be powerful for controlling more complex processes, for which no mathematical formulation of parameter relations exists. The possibility exists to "tune" the controller for better performance by adapting the membership functions. An expert can do this via a simple interface.

\section{The application: Conditioning of High Voltage RF cavities}

For certain processes the control strategy can be easily expressed in rules. The problem of conditioning high voltage radio frequency (RF) cavities is typically an application for which we can formulate the control strategy with rules and for which we need gradual changes of the control values (the more the more. Important for the choice of the prototype application was also that a conventional program existed already for comparison, and that the application had a small number of rules and actions.

The problem is the following. The electric field that is needed for operation cannot directly be applied to the RF cavity without breakdowns of the field, so-called flashovers. The occurrence of flashovers has to be carefully controlled; some of them are useful to clean the cavity surface, too many might damage the cavity. The increase of the electric field has to be done in small steps if a few flashovers occur, in bigger steps if no flashover occurs and be decreased if too many flashovers occur. Instead of counting flashovers, the vacuum can be used as an indicator of the level of conditioning, since there is an increase of pressure if a flashover occurs. We give examples for the case where the flashovers are used as input parameter.

The input parameter asked from the user of the program is the final level of the electric field. At each control cycle the number of flashovers and the actual level of the electric voltage applied to the cavity are read. Some internal parameters are used, for example the number of consecutive flashovers and the highest voltage achieved.

\section{Modelling with Fuzzy Logic}

The kernel of the control program is a small rule base. The control actions are easily expressed in rules for this application. They are very general and apply for many different pieces of equipment of the same type. As an example, some of them are shown in figure 1 .

The main idea is to unite a set of numerical values (a range) under one unique linguistic term. For example, the set of numerical values that correspond to "flashovers many" associated with the set of numerical values corresponding to "a small difference between the actual and the final voltage achieved" make the rule 1 in the 
example, fire (it is activated). This means that a whole set of control values corresponding to "small amount" are possible as a consequence. In this way we can cover a wide amount of equipment with more or less the same behavior with the same rule base. However, we need to convert the numerical input value (for this application we suppose numerical input data) to the linguistic labels "not many", and the output value "small amount" has to be converted to a numeric output value for being able to perform control. This is done in a software module in the control program kept apart from the rules, ensuring this quantification by using fuzzy logic.

Rule 1: IF nashovers are not many

AND IF the difference between the actual level and the final level is small

THEN the increase in the RF power is small

Rule 2: IF previous flashovers are not many

AND IF the difference between the maximal level obtained and the actual level is high THEN the increase in the RF power is large

Rule 3: IF previous flashovers are not many

AND IF the difference between the final level and the actual level is high

THEN the increase in the RF power is medium

Rule 4: IF flashovers are not too many

THEN the decrease in the RF power is medium

Fig. 1 : Example of expert knowledge in form of rules for the High Voltage Conditioning of RF Cavities. This is the whole rule base for the application. There are in addition a few rules for program control, not shown in the figure.

Suppose we have a parameter "number of flashovers" with two characterizations (linguistic labels): "toomany-flashovers" and "many-flashovers". We have to be able to convert a numerical value, let's say 75 , to one of the labels to be able to use it in the rules.

Each label is represented by a mathematical function, called membership function (denoted by $\mathrm{f}$ ), which takes real values from 0 to 1 . It is unique to the specific equipment. For the two labels for the parameter "number of flashovers", the membership functions of the labels "few", "many" and "too many" look like as in figure 2.

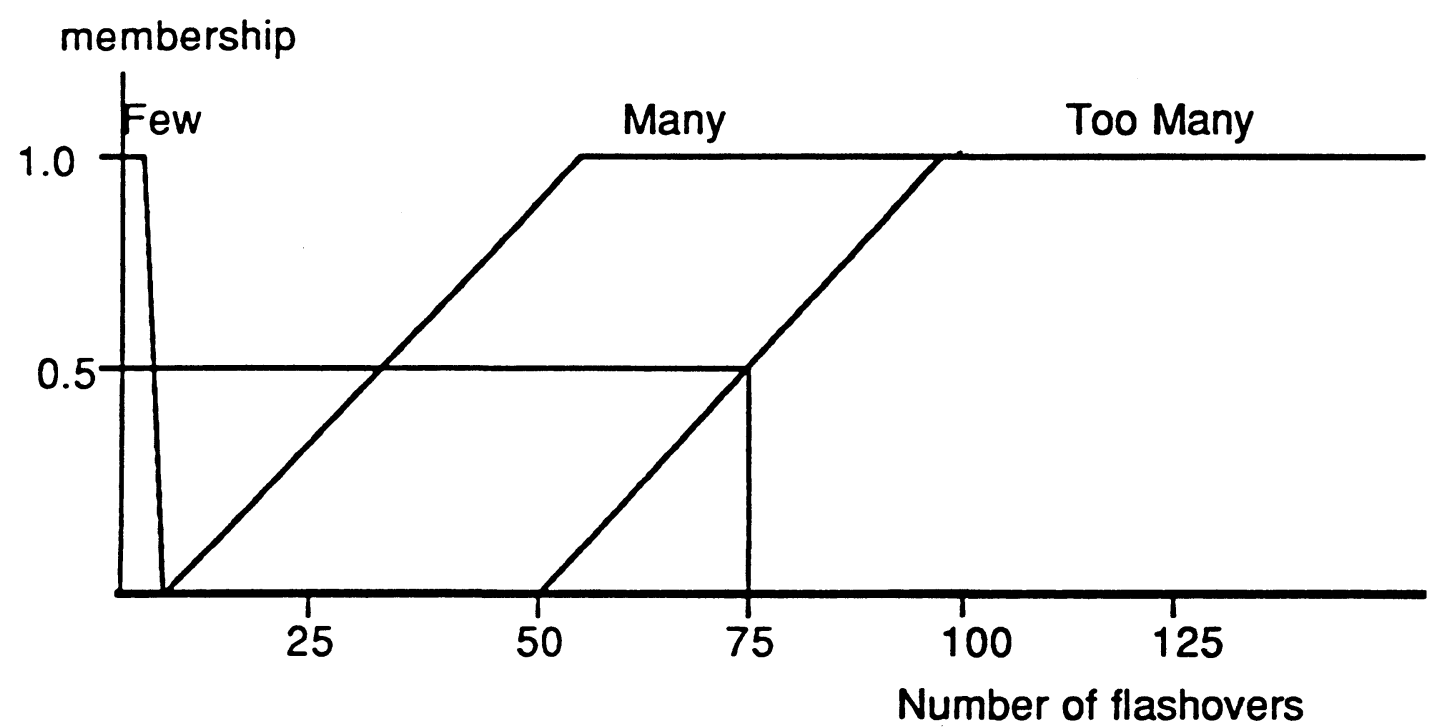

Figure 2. Example of membership functions for the characterizations of the parameter "number of flashovers". 
Looking at this figure we conclude that 75 flashovers corresponds to "many-flashovers" but to the degree 0.5 . The value 75 does not at all correspond to the label "few" since the membership function is zero. Now we have fuzzified the value 75 and the premise "IF the number of flashovers is many-flashovers" will be considered as somewhat true, level 0.5 .

Fuzzy rules fire to a certain degree depending on the level of each antecedent in the premise of the rule. The antecedents are evaluated using the membership functions to produce values which are then combined using fuzzy operators to produce the final output activation level of the rule. In our case we use the product of the membership functions for combining the premises. The fuzzy set for the output is then modified using this activation level according to some fuzzy implication operator that has to be chosen to fit the application. Important in a control application is to choose an operator that gives continuity for the output parameters i.e. the more the measurement corresponds to the label in the premise the more the output corresponds to the label in the conclusion.

\section{The Implementation}

\subsection{The layout of the program}

The main objective for the implementation is to separate the general knowledge for this type of process i.e. knowledge that holds for many cavities. The knowledge describing the particularities of the cavities is kept apart. To do this, there is a rule base containing the general information and a little data base contains the description of the particular cavities. There is a small library of routines to administrate the treatment and conversion of the numeric information to the linguistic terms; which we call "Generalized Modus Ponens" treatment which uses the data base. This database, in the form of a file containing two values per label and per parameter, namely the top and the bottom of the piece wise linear membership function, constitutes the "model". In this way the model for each process reduces to only a collection of real number pairs, which later can be edited by a simple user interface. See fig 3.

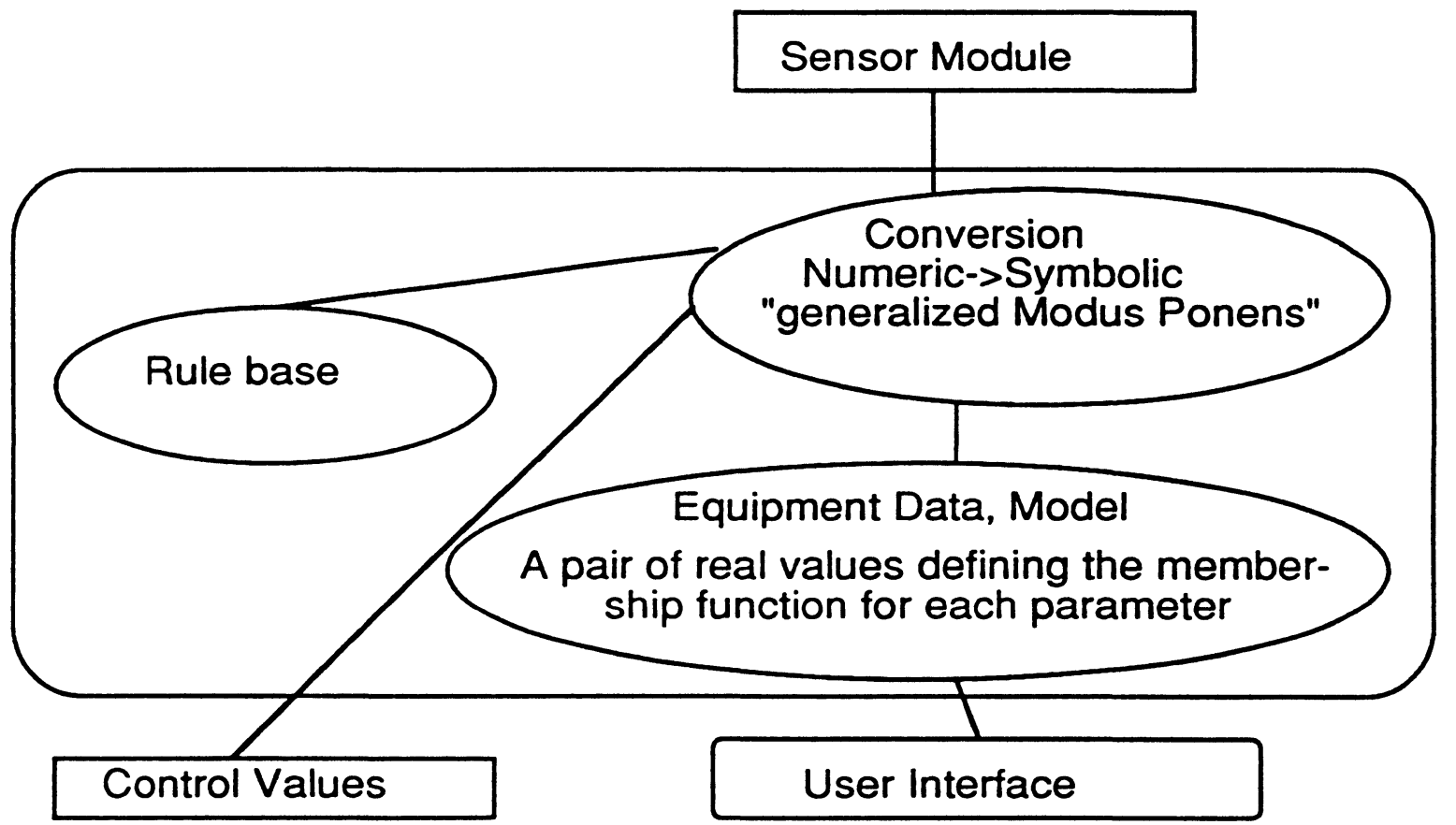

Figure 3. Layout of the program for Conditioning of High Voltage Cavities 
The rules are implemented in OPS5. There are about ten rules. Apart from the rules describing the control behaviour we also need rules for program control, like data acquisition. The rules are executed only with linguistic data, so we need another part to convert numerical data into linguistic data. This is called generalized Modus Ponens.

\subsection{The Generalized Modus Ponens module}

The classical deduction, Modus Ponens, has to be generalized to work with fuzzy logic. Modus Ponens has the form: from data $\mathrm{P} 1, \ldots, \mathrm{Pn}$ and a rule "IF $\mathrm{P} 1, \ldots ., \mathrm{Pn}$ THEN $\mathrm{Q}^{n}$ one is able to conclude $\mathrm{Q}$. P1,..., $\mathrm{Pn}$ are measurements and $Q$ is a proposed modification of the control value. This deduction law is now generalized in the following way: consider $X$ and $Y$ as variables, and $A$ and $B$ as labels for the variables $X$ and $Y$. $A^{\prime}$ and $B^{\prime}$ are numeric values for $X$ and $Y$. From the fact $X$ is $A^{\prime}$ (e.g. the number of flashovers is 75), and a rule

\section{IF X IS A, THEN Y IS B}

e.g. if the flashovers $(X)$ are many $(A)$ then the modification of power $(Y)$ is decreased $(B)$, one concludes, using generalized Modus Ponens, that

$\mathrm{Y}$ is $\mathrm{B}^{\prime}$

e.g. the modification of the electrical voltage $(Y)$ is a decrease by $10 \mathrm{~kW}(B)$. The conclusion for the generalized Modus Ponens depends on the fact and also on the rule. In this application a particular relation is used, that is:

$$
g_{B}(B)=f_{A}(A) \text {. }
$$

where $\mathrm{g}_{\mathrm{B}}$ is the membership function for the label $\mathrm{B}$ in the conclusion.

Other ways of choosing the relation for calculating the output value from the input value exist. See ref. [ ]. But this simple relation is satisfactory for our application. It fulfills the important criterion of continuity. By continuity we mean here that we can say "the more $\mathrm{X}$ is $\mathrm{A}$ the more (less) $\mathrm{Y}$ is $\mathrm{B}^{\text {". }}$.

The output value can now be calculated to

$$
B^{\prime}=g_{B}{ }^{-1}\left[f_{A}\left(A^{\prime}\right)\right]
$$

where $f_{A}\left(A^{\prime}\right)$ is the membership degree of $A^{\prime}$ for the left hand side label $A$ used in the rule given above, and $g_{B}\left(B^{\prime}\right)$ the membership degree of the right hand side label $B$ of the rule.

In some rules a conjunction of premises is used. We have to chose an operator for conjunction that fulfills a number of criteria. Two important criteria for the conjunction are that the resulting membership function should be between 0 and 1 and that the resulting membership degree increases if one of the membership degrees of the premises increases. The product suits us well. Therefore we get for the output value

$$
B^{\prime}=g_{B}{ }^{-1}\left[f_{A_{1}}\left(A_{1}\right) * f_{A_{2}}\left(A_{2}\right)^{*} \ldots{ }^{*} f_{A_{n}}\left(A_{n}\right)\right]
$$

\subsection{Calculation of the membership degrees and the final output value}

$$
\begin{aligned}
& f_{A}\left(A^{\prime}\right)=0 \quad \begin{array}{l}
\text { if } A^{\prime} \leq \text { base } A \\
\left(\text { or } A^{\prime} \geq \text { base } A \text { if } f_{A}\right. \text { is decreasing) }
\end{array} \\
& =1 \quad \begin{array}{l}
\text { if } A^{\prime}>\text { top } \\
\left(\text { or } A^{\prime} \leq \text { top } A \text { if } f_{A}\right. \text { is decreasing) }
\end{array} \\
& =\text { (base } A-A) /(\text { base } A \text {-top } A \text { ) otherwise. }
\end{aligned}
$$


Using relation (1)we get for the final calculation of the output of the controller

$$
\begin{aligned}
& B^{\prime}=\left\{\left[f_{A_{1}}\left(A_{1}\right) * f_{A_{2}}\left(A_{2}\right)^{*} \ldots * f_{A_{n}}\left(A_{n}\right)\right]^{*}\right. \\
& \text { (top B-base B) + base B }
\end{aligned}
$$

\subsection{Characterizing the piece of equipment}

To get a good control behaviour the membership functions have to be defined correctly. Since the equipment specialist has formulated the rules, he has a feeling for the labels he has chosen to describe the process. For example, if he uses "many flashovers" in a rule, he can certainly quantify what he means by "many flashovers". In our application, the specialist has to give the top and the bottom of the membership function for all the labels used, i.e. between which two values the label "many flashover" holds. This gives us a very simple "database" of pairs that describe the different particularities of the different cavities. If the specialist finds that the model is not completely satisfactory, he can tune the behaviour (offline) by changing the top and the base values for the labels.

\section{Status}

The behaviour of the module has been compared to the behaviour of the classical control program for the for the RF tanks of the LINAC II ( $50 \mathrm{MeV}$ ) of the CERN PS. The comparison has been made in the following way. Input/output values from the classical control program have been recorded and the input values have been fed to the fuzzy control module. The output results from the fuzzy control module have been compared to the recorded outputs. The result is encourageing and the program will in a near future be tested for the LINACII RFQ.

The membership functions for the labels are for the moment on a file. An simple editor of this file will be written so that the specialist can une his equipment in an easy way. We envisage automatic tuning of these membership functions. The difficulty is to define the criterion for this tuning and to express it in such a way that it can be used by an automatic tuning program.

\section{Discussion}

This very simple example of the use of fuzzy logic for control shows that fuzzy logic could be a good way of approaching closed loop control for processes if they can be conveniently expressed in rules. Many control loops can be expressed in if-then statements in a traditional programming language. However, if there are many rules and complex logic, and where the objects have many attributes, it is an advantage to use a rule based tool. The advantages are the simplicity and the high modularity of the implementation. The model can be adapted in an easy way by the user of the control program. There is an interesting possibility to let this adaptation be done by an automatic procedure. For doing this we need to investigate which criteria define this optimization.

Rule based programming needs experts and this might be a problem for continuing the development. However, tools now exist on the market that give the basic bricks for the implementation of fuzyy logic controllers. Using these tools we can continue the investigations and concentrate on the optimization criteria and the process modelling.

6. Acknowledgments

We want to thank J Fuchs (CERN) and B Bouchon-Meunier (LAFORIA) for advice in this project. We are also indebted to G. Rossat (CERN), who gave us the opportunity to participate in the RF conditioning of LIPS 01 (CERN).

\section{References}

[1] B. Bouchon-Meunier, "Management of Uncertainty in Knowledge-based Systems", in Encyclopaedia of Computer Science and Technology, Vol. 20 supp. 5; Holzmann A.G., Kent A, Williams J.G. (Eds), Marcel Dekker Publisher, pp. 327-337.

(2) E. Trillias, L. Valverde, "On Mode and Implication in Approximate Reasoning", in Approximate Reasoning in Expert Systems; Gupta M.M., Kandel A., Bandler W., Kuszka J.B. /Eds), Elsevier Science Publishers, pp. 157-166.

(3) L. Zadeh, "The Role of Fuzzy Logic in the Management of Uncertainty in Expert Systems"in Fuzzy Sets and Systems; Zimmermann H.J. /Ed.), North-Holland Publisher, Vol. 11, pp. 199-227. 